\title{
A Comprehensive Review of Arsenic Exposure and Risk from Rice and a Risk Assessment among a Cohort of Adolescents in Kunming, China
}

\author{
Noelle Liao ${ }^{1}$, Edmund Seto ${ }^{2}$, Brenda Eskenazi ${ }^{3}$, May Wang ${ }^{4}$, Yan Li ${ }^{5}$ and Jenna Hua ${ }^{6} *$ (i) \\ 1 Department of Preventive Medicine, Keck School of Medicine, University of Southern California, \\ Los Angeles, CA 90033, USA; noelleliao@alumni.usc.edu \\ 2 Department of Environmental \& Occupational Health Sciences, School of Public Health, University of \\ Washington, Seattle, WA 98195, USA; eseto@uw.edu \\ 3 Department of Maternal and Child Health, School of Public Health, University of California, \\ Berkeley, CA 94720, USA; eskenazi@berkeley.edu \\ 4 Department of Community Health Sciences, School of Public Health, University of California, \\ Los Angeles, CA 90095, USA; maywang@ucla.edu \\ 5 Kunming Medical University, Kunming 650221, Yunnan, China; yanli20021965@21cn.com \\ 6 Stanford Prevention Research Center, School of Medicine, Stanford University, Stanford, CA 94305, USA \\ * Correspondence: jenna_hua@stanford.edu; Tel.: +1-650-723-6254
}

Received: 1 August 2018; Accepted: 3 September 2018; Published: 8 October 2018

\begin{abstract}
Inorganic arsenic (iAs) is carcinogenic and highly concentrated in rice. Dietary exposure to iAs is concerning among adolescents due to their developmental stage and iAs's long-latency effects. This paper aimed to assess iAs exposure from rice and related lifetime cancer risks (LCR) among adolescents in Kunming, China. A comprehensive literature review of iAs levels in rice and LCR in humans was also conducted. Average daily consumption of rice (ADC) was estimated from 267 adolescents (15-18 years). Rice samples obtained from 6 markets were analyzed for iAs concentration (AC). Estimated daily intake (EDI) of iAs was calculated using ADC, AC, and average body weight (BW). Lifetime Cancer Risk (LCR) was calculated using EDI and U.S. EPA derived iAs oral slope factor. The AC was $0.058 \mathrm{mg} / \mathrm{kg}$ and the average BW and ADC were $67.5 \mathrm{~kg}$ and $410 \mathrm{~g} / \mathrm{day}$ for males and $55.5 \mathrm{~kg}$ and $337 \mathrm{~g} /$ day for females. The EDI and LCR were $3.52 \times 10^{-4} \mathrm{mg} / \mathrm{kg}-\mathrm{BW} / \mathrm{day}$ and $5.28 \times 10^{-4}$ for both males and females, with LCR 5 times above the U.S. LCR upper limit of $1.0 \times 10^{-4}$. While the AC was below the Chinese maximum contaminant level of $0.2 \mathrm{mg} / \mathrm{kg}$, study results indicated that Kunming adolescents may be at increased risk for iAs-related cancers.
\end{abstract}

Keywords: arsenic; cancer; rice; concentration; diet; risk assessment; exposure; China; Asia; adolescents

\section{Introduction}

Exposure to toxic contaminants in food is presumed to be one of the major public health challenges for the 21st century [1]. Arsenic (As) is especially prevalent in the environment and can easily enter the food system through contaminated soil or water. Of arsenic's two chemical forms (organic and inorganic), inorganic arsenic (iAs) is classified by the International Agency for Research on Cancer (IARC) as a non-threshold (even small doses may provide some cancer risk) class 1 human carcinogen and is associated with skin, lung, liver, kidney, and bladder cancers [2]. Among As species, i-AsIII (arsenite) is the most abundant species found in rice and also the most toxic to humans [3,4].

Arsenic has no single major mode of action in the human body [5]. A few of the well-documented mechanisms describing arsenic's influence on cancer are (1) increasing the generation of hydrogen peroxide and superoxide anions; (2) interacting with cysteine residues in zinc finger domains, 
ultimately leading to loss of protein function; (3) deregulating cell proliferation and inducing epigenetic alterations [6]. Because cancer risk may be non-linear, a safe level cannot be determined by extrapolating risk from high dose exposures [6].

Flooded rice fields and the anaerobic nature of paddy soils facilitate the buildup of arsenic in the rice crop compared to other agricultural crops [2]. Rice can accrue up to 10-20 times more arsenic than wheat or barley because silica and phosphate transporters in the rice crop effectively move iAs up into the rice grain [7]. According to the Food and Agriculture Organization of the United States (FAO), China is the highest rice producing and consuming country in the world and accounts for a third of the global supply of rice [8,9]. Rice grown in Asia has also been found to have higher iAs content than rice grown in the U.S. [10]. An estimated $57-96 \%$ of the total arsenic measured in Chinese-produced rice has been found to be of the inorganic form, which stresses the importance of conducting iAs risk assessments in China [11]. Nonetheless, estimating iAs exposure from rice is difficult in China due to the vast size of the country, its variable geology, and the country's diverse dietary patterns among sub-populations [11]. These factors strongly emphasize the importance of doing regional risk assessment studies in China.

Until recently, official food monitoring across the world considered only total As in rice (not inorganic As species) and lately iAs exposure and its health effects have become national and international concerns [12]. Likewise, many studies rarely measure iAs in rice directly and instead only estimate iAs using a fraction of the total arsenic measured [13]. Related studies also lack the use of individual-level data and/or do not estimate cancer risk using rice samples, individual consumption rates, and body weights from the same, specific region. These factors are all critical for an accurate health risk assessment of iAs. Furthermore, certain populations may be particularly at risk. For example, because of the long latency period (approximately 25 years) of iAs-related cancer, children have a greater potential for long-term exposure [14]. Children, including adolescents, are also in a critical window of development, may have a high calorie per unit body weight diet compared to adults, and tend to be more exposed to contaminants unique to specific foods due to selective and less diverse dietary patterns [15].

Therefore, the aims of this study are to: (1) conduct a comprehensive literature review of recent studies published after 2010 that measured arsenic concentrations in rice and/or arsenic exposure levels in humans, or conducted arsenic-related cancer risk assessments; (2) estimate lifetime cancer risk among a cohort of adolescents living in Kunming, China using directly measured iAs levels in locally sampled rice and individual-level rice consumption data of Kunming adolescents; and (3) compare this study cohort's iAs exposure rates and lifetime cancer risks to that of other populations in the published literature.

\section{Materials and Methods}

\subsection{Literature Review}

A comprehensive literature review was conducted using Google Scholar and PubMed with key search terms including arsenic, rice, concentration, cancer, exposure, diet, risk assessment, China, Asia, and adolescents. Papers excluded in this manuscript's tables were reviews, papers published before 2010 in order to provide a more concise review of more current As levels in rice and exposure levels in populations, and arsenic risk assessments with outcomes other than cancer. However, papers with an outcome other than cancer that had measurements of arsenic concentrations in rice, as well as having China or Asia as the study locations, were included in the assessment in order to account for those Asian risk assessment studies that measured As levels in rice.

\subsection{Rice Sample Collection and Lab Analysis}

Short-grain white rice samples were collected from six Kunming markets, varying by location and market type, to represent foods purchased in different Kunming neighborhoods and by different 
financial preferences, such as from a high-end market selling organic food and at a Carrefour (hypermarket). The collected rice samples were sourced from growing regions in Northern China, as is most of the rice sold in Kunming.

Samples were sent to the commercial laboratory Merieux Nutrisciences located in Ningbo, China for analysis. The laboratory received seven labeled samples from seven different locations, two of which were from the same location in order to assess the reliability of the lab's measurement procedures. The lab used the 2009 updated version of the GB/T 5009.11-2003 method for determination of abio-arsenic (iAs) in the received rice samples. By means of hydride generation atomic fluorescence spectrophotometry, iAs was extracted in the form of chloride and separated from organic arsenic during water bathing with $\mathrm{HCL}$, and its concentration was measured [16]. Specifically, $2.50 \mathrm{~g}$ of solid sample was blended with $20 \mathrm{~mL}$ of HCL $(1+1)$ solution and put into a $60{ }^{\circ} \mathrm{C}$ water bath for $18 \mathrm{~h}$ and then filtered. $4 \mathrm{~mL}$ of filtrate was mixed with $1 \mathrm{~mL}$ of KI-thiourea mixed solution and 8 drops of $n$-octanol, diluted to volume with water, and settled for $10 \mathrm{~min}$ to determine iAs concentration. Standard series of iAs determination used $1 \mu \mathrm{g} / \mathrm{mL}$ As3+ standard solution [16].

\subsection{Study Population}

Adolescents (267, 45.7\% males and 54.3\% females) aged 15-18 years old and all of Han ethnicity were recruited from two local Kunming high schools in 2015 using convenience sampling methods. The schools had a wide catchment of students who lived in various regions of the city. Study participants completed 72-h dietary recalls which captured their diets over two weekdays and a weekend day (Thursday, Friday and Saturday). Participants were trained on estimating portion sizes and how to fill out a 72-h dietary recall. Participants' weights were also measured in light clothing without shoes to the nearest $0.1 \mathrm{~km}$ using an electronic scale. All human subject data collection procedures were approved by the Institutional Review Boards of UC Berkeley and Kunming Medical University (2014-03-6097).

\subsection{Exposure Estimation and Cancer Risk Calculation}

Average daily consumption rates (ADC) of white rice (g/day) were estimated using the 72-h recalls for both males and females separately. Estimated daily intake (EDI) of iAs from rice consumption (mg/kg-BW/ day) was calculated using the ADC, average concentration (AC) of iAs in sampled rice, and average body weight of the adolescents (BW) [17]:

Estimated daily intake:

$$
\mathrm{EDI}=(\mathrm{AC} \times \mathrm{ADC}) / \mathrm{BW},
$$

Lifetime Cancer Risk (LCR), the probability of excess lifetime cancer risk, was calculated using the EDI and the U.S. EPA derived iAs oral slope factor (SF), $1.5(\mathrm{mg} / \mathrm{kg}) /$ day [5]. The oral slope factor is the plausible upper-bound estimate of the probability of a response per unit intake of a chemical over a lifetime and refers to a linear, non-threshold model of risk [7]. Lifetime cancer risk assumes daily exposure (365 days of the year) over one's entire lifetime.

Lifetime cancer risk:

$$
\mathrm{LCR}=\mathrm{EDI} \times \mathrm{SF},
$$

Stata version 15.0 (StataCorp, College Station, TX, USA, 2017) was used to calculate the average daily consumption rates of rice and mean body weights of males and females in the study cohort. A $t$-test was conducted to compare the ADCs and BWs between males and females. A $p$-value of less than or equal to 0.05 was selected to indicate statistical significance. 


\section{Results}

\subsection{Literature Review}

Fifty four papers were screened and 38 fitted the table inclusion criteria. Only seven studies focused on Southern China and the other eight Chinese studies focused on other regions or multiple provinces throughout China. It was estimated that Southern China has 2.5 times higher rice consumption rates than Northern China and higher concentrations of arsenic in rice [11,18]. Of those seven studies focusing on Southern China, five studies had separate risk assessment analyses for children and one study for adolescents. Thirteen out of the 38 studies included in the review tables (Tables 1 and 2) directly measured iAs in rice. Huang et al. conducted the only regional risk assessments in China using individual-level data [19]. The other studies conducted in China pulled consumption data from previously published literature or used nation-wide dietary or ecological-level data. Given the varied dietary patterns among different regions of China and among different age groups, using averaged nation-wide data may not be reflective of unique regional differences.

Table 1 summarizes the studies that assessed arsenic concentrations (total As and/or iAs) in rice and did not include a cancer risk assessment. Chen et al. tested 160 rice samples from local markets in 20 provinces in China and the results indicated average iAs levels $(\mathrm{mg} / \mathrm{kg})$ of 0.054 across all regions, 0.058 in Southern China, 0.61 in Middle China, 0.042 in Eastern China, and 0.048 in Northern China [20]. Table 2 summarizes the studies that conducted cancer risk assessments of arsenic. Some of the arsenic risk assessment studies do not calculate LCR and instead compare their population's EDI to the previous provisional tolerable daily intake (PTDI) of $2.1 \times 10^{-3} \mathrm{mg} / \mathrm{kg}$-BW/day recommended by the WHO [21]. Notably, the PTDI has been withdrawn because it is no longer considered health protective due to the $\mathrm{BMDL}_{0.5}$ of $3.0 \mu \mathrm{g} / \mathrm{kg}-\mathrm{BW} /$ day (lower $95 \%$ confidence limit on the benchmark dose for a $0.5 \%$ increased incidence of lung cancer) residing in its range $\left(2 \times 10^{-3}-7 \times 10^{-3} \mathrm{mg} / \mathrm{kg}-\mathrm{BW} /\right.$ day $)$ [10]. The benchmark dose is the dose that is associated with a specific change in an adverse response compared to the response in unexposed persons [22]. We note that if data on adolescents were specifically provided in a study, we presented those in the table. As indicated in Table 2, iAs exposures and related LCRs were particularly high for Asian countries such as China, India, Pakistan, Bangladesh and Malaysia and lower for Europe and the Americas, with adolescents in Bangladesh having exceptionally high LCRs (Figure 1). Figure 1 compares LCR values in the risk assessment studies (Table 2) that reported LCRs. It serves as a graphical demonstration of the diversity of cancer risks and the populations that are potentially most at risk. The U.S. EPA LCR acceptable upper limit is $1.0 \times 10^{-4}$ and it is apparent that many Asian countries and even adolescents in those populations greatly exceeded the upper limit [5].

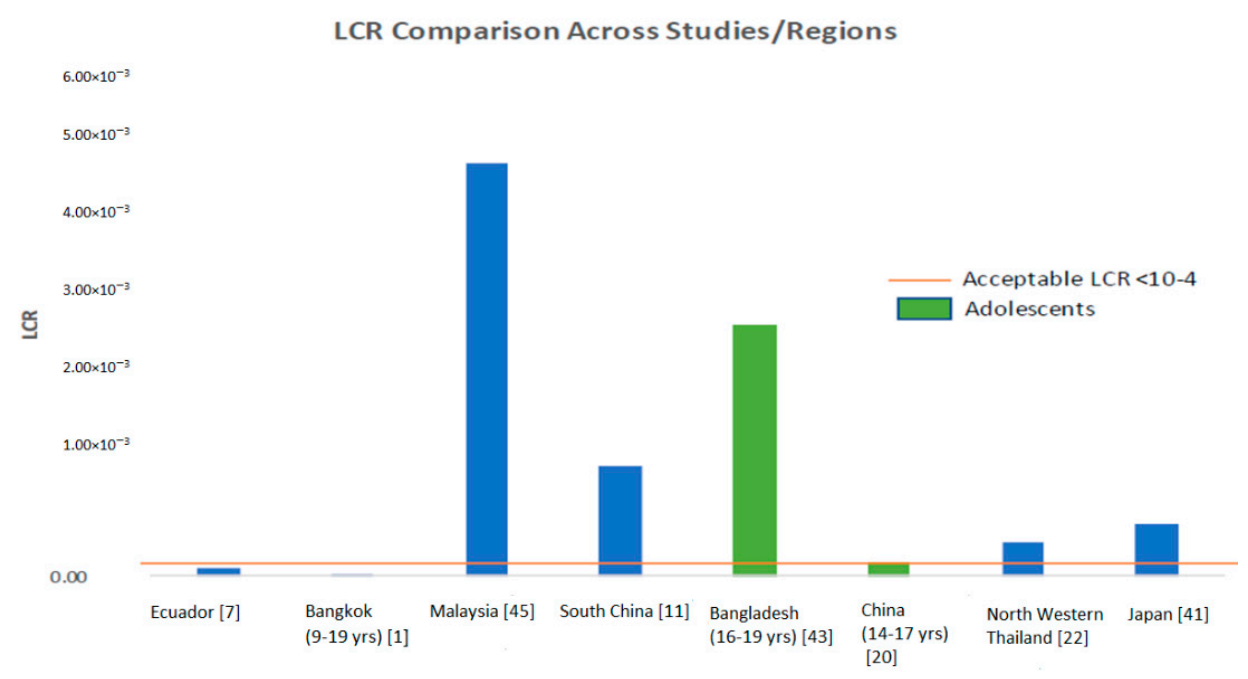

Figure 1. Comparison of LCR values from different studies and regions. 
Table 1. Summary of studies assessing arsenic concentrations in rice.

\begin{tabular}{|c|c|c|c|c|}
\hline Study Location & Author(s) and Year & Source of Rice & Type of Arsenic Measured & Mean $(\mathrm{mg} / \mathrm{kg}) \pm \mathrm{SD}$ (Range) \\
\hline USA & He et al. 2010 [23] & Samples purchased in New York & Total As & $0.14 \pm 0.0050$ \\
\hline Japan & Narukawa et al. 2012 [24] & 20 samples from all over Japan & iAs & $0.10(0.056-0.20)$ \\
\hline Bangladesh, China, USA & Norton et al. 2012 [25] & 6 field trials & Total As & $\begin{array}{c}\text { Faridpur: } 0.44(0.19-0.90) \\
\text { Qiyang: } 0.68(0.36-1.27) \\
\text { Arkansas (2006): } 0.38(0.10-0.99) \\
\text { Arkansas (2007): } 0.25(0.030-1.040) \\
\text { Texas (flooded): } 0.63(0.17-1.68) \\
\text { Texas (non-flooded): } 0.045(0.0090-0.13)\end{array}$ \\
\hline China & Sun et al. 2012 [26] & $\begin{array}{l}2 \text { samples from market in Guangzhou and rice field in } \\
\text { Hunan Province }\end{array}$ & iAs & $\begin{array}{c}0.35 \pm 0.0060 \\
(0.40-0.29)\end{array}$ \\
\hline China & Dai et al. 2014 [27] & 108 samples from local markets & iAs & $\begin{array}{c}\text { Jiangsu: } 0.063 \\
\text { Jiangxi: } 0.057 \\
\text { Zhejiang: } 0.059 \\
\text { Mean of all: } 0.059 \\
\text { Range of all: }(0.027-0.098)\end{array}$ \\
\hline China & Fang et al. 2014 [18] & 92 samples from fields of main rice-growing provinces & Total As & $\begin{array}{c}\frac{\text { Northern China }}{0.050 \pm 0.040} \\
\left({ }^{*} \mathrm{ND}-0.13\right) \\
\text { Southern China } \\
0.11 \pm 0.050 \\
\left({ }^{*} \mathrm{ND}-0.14\right)\end{array}$ \\
\hline Bangladesh & Ahmed et al. 2016 [21] & 10 market samples & Total As & $\begin{array}{l}0.32 \pm 0.16 \\
(0.14-0.43)\end{array}$ \\
\hline China & Chen et al. 2018 [20] & 160 samples from local markets in 20 provinces & iAs & $\begin{array}{c}0.054 \\
(0.0090-0.13)\end{array}$ \\
\hline
\end{tabular}

${ }^{*} \mathrm{ND}=$ not detectable 
Table 2. Summary of arsenic exposure levels and arsenic-related cancer risk assessments.

\begin{tabular}{|c|c|c|c|c|c|c|c|c|}
\hline $\begin{array}{c}\text { Study } \\
\text { Location }\end{array}$ & $\begin{array}{l}\text { Author(s) } \\
\text { and Year }\end{array}$ & $\begin{array}{l}\text { Age/Group } \\
(N=)\end{array}$ & Source of Arsenic & Type of Arsenic & $\begin{array}{c}\text { Mean }(\mathrm{mg} / \mathrm{kg}) \\
\text { (Range) }\end{array}$ & $\begin{array}{c}\text { As Exposure Estimation } \\
\text { (IR = Ingestion Rate of Rice) }\end{array}$ & EDI (mg/kg-BW/Day) & Cancer Risk \\
\hline China & $\begin{array}{l}\text { Liang et al. } \\
2010[28]\end{array}$ & - & $\begin{array}{l}21 \text { rice samples } \\
\text { from } 13 \text { provinces }\end{array}$ & iAs & $0.082(0.049-0.22)$ & $\begin{array}{c}\mathrm{IR}=550 \mathrm{~g} / \text { day (reported from } \\
\text { Zhu et al. 2008b) } \\
\text { Assuming BW }=60 \mathrm{~kg}\end{array}$ & $0.045 \mathrm{mg} /$ day & $\begin{array}{l}\text { 37.6\% contribution to the MTDI } \\
\text { (also }{ }^{2} \text { PTDI) }\end{array}$ \\
\hline $\begin{array}{l}\text { Southern } \\
\text { China }\end{array}$ & $\begin{array}{l}\text { Liu et al. } \\
2010[13]\end{array}$ & $\begin{array}{l}136 \text { hair \& } 61 \\
\text { urine samples }\end{array}$ & $\begin{array}{l}33 \text { brown rice } \\
\text { samples from } \\
\text { Lianhuashan } \\
\text { tungsten } \\
\text { mining area }\end{array}$ & $\begin{array}{l}\text { Total As (iAs } \\
\text { estimated using } \\
83 \% \text { of total As) }\end{array}$ & $\begin{array}{l}\text { Total As: } 0.56 \\
(0.15-1.09)\end{array}$ & $\begin{array}{l}\text { (IRs from Khan et al. } 2008 \text { and } \\
\text { Wang et al. 2005) } \\
\text { Adult IR }=491.5 \mathrm{~g} / \text { day } \\
\text { Assuming Adult BW }=60 \mathrm{~kg} \\
\text { Children (1-14 years) IR }=289.6 \text {, } \\
\text { 232, 92.6 g/day }\end{array}$ & Adults: $0.23 \mathrm{mg} /$ day & EDI $>^{2}$ PTDI \\
\hline India & $\begin{array}{l}\text { Mondal et al. } \\
2010[29]\end{array}$ & $\begin{array}{c}(N=232) \text { from } \\
3 \text { different } \\
\text { villages }\end{array}$ & $\begin{array}{l}\text { Drinking water, } \\
\text { cooking water, raw } \\
\text { rice, \& cooked rice } \\
\text { from households }\end{array}$ & Total As & $\begin{array}{c}\text { Raw rice } \\
0.12 \pm 0.090 \\
0.16 \pm 0.050 \\
0.12 \pm 0.020 \\
\text { Drinking water }(\mu \mathrm{g} / \mathrm{L}) \\
130 \pm 128 \\
40 \pm 99 \\
1.0\end{array}$ & $\begin{array}{c}\text { Water intake } \\
\text { Males: } 3.1 \pm 1.0 \mathrm{~L} / \text { day } \\
\text { Females: } 2.6 \pm 0.9 \mathrm{~L} / \text { day } \\
\text { Rice IR from National Database } \\
\text { of NNMB }(2002) \\
\text { Males: } 11.60 \mathrm{~g} / \mathrm{kg} / \text { day } \\
\text { Females: } 11.27 \mathrm{~g} / \mathrm{kg} / \text { day }\end{array}$ & $\begin{array}{c}\text { Water } \\
\text { Medians: } 2.0 \times 10^{-5} \\
7.7 \times 10^{-4}, 2.03 \times 10^{-3} \\
\text { Cooked rice } \\
\text { Medians: } \\
3.0 \times 10^{-4}, 5.0 \times 10^{-4} \\
8.4 \times 10^{-4}\end{array}$ & $\begin{array}{l}\text { (Risk based on total exposure } \\
\text { from drinking water, rice, } \\
\text { and cooking of rice) } \\
\text { LCR } \\
\text { Bhawangola-I block: } 4.35 \times 10^{-3} \\
\text { Chakdha block: } 2.04 \times 10^{-3} \\
\text { Khejuir-I block: } 4.56 \times 10^{-4}\end{array}$ \\
\hline Ghana & $\begin{array}{l}\text { Adomako et } \\
\text { al. } 2011[30]\end{array}$ & $\begin{array}{l}\text { Ages } 12 \text { to } \\
>40 \text { years } \\
(N=204)\end{array}$ & $\begin{array}{l}549 \text { wholegrain rice } \\
\text { samples from } \\
\text { Ghana, USA, EU, } \\
\text { and Asia }\end{array}$ & $\begin{array}{l}\text { Total As } \\
\text { (iAs estimated } \\
\text { using } 82.9 \% \text { of } \\
\text { total As) }\end{array}$ & $\begin{array}{c}\text { Total As } \\
\text { Global mean: } 0.14 \\
\text { Ghana: } 0.11 \pm 0.02 \\
\text { USA: } 0.22 \pm 0.010 \\
\text { iAs } \\
\text { Ghana: } 0.091 \\
\text { USA: } 0.092 \\
\text { Thailand: } 0.10\end{array}$ & $\begin{array}{c}\text { Ghana IR } \leq 33.2 \text { to }>232.2 \mathrm{~g} / \text { day } \\
\text { Baseline IR }=99.6 \mathrm{~g} / \text { day, } \\
\text { rounded to } 100 \mathrm{~g} / \text { day of dry rice } \\
\text { Average } \mathrm{BW}=60 \mathrm{~kg}\end{array}$ & $\begin{array}{l}\text { Ghana: } 9.1 \mathrm{~g} / \text { day } \\
\text { USA: } 9.2 \mathrm{~g} / \text { day } \\
\text { Thailand: } 10.1 \mathrm{~g} / \text { day }\end{array}$ & $\begin{array}{l}\text { Used cancer slope factor of } 3.67 \\
\text { mg/kg/day (cited by } \\
\text { Tsuji et al. 2007): } \\
\text { LCR } \\
\text { Ghana: } 5.57 \times 10^{-4} \\
\text { USA: } 5.6 \times 10^{-4} \\
\text { Thailand: } 6.2 \times 10^{-4}\end{array}$ \\
\hline China & $\begin{array}{l}\text { G. Li et al. } \\
2011[11]\end{array}$ & $\begin{array}{c}\text { Adults } \\
(N=68,962)\end{array}$ & 494 rice samples & $\begin{array}{l}\text { iAs } \\
\text { (obtained from } \\
\text { regression } \\
\text { equation) }\end{array}$ & $\begin{array}{c}\text { North } \\
0.092 \pm 0.020 \\
\text { South } \\
0.099 \pm 0.042\end{array}$ & $\begin{array}{c}\text { (Data from China National } \\
\text { Nutrition and Health Survey } \\
\text { (CNNHS) } \\
\text { North } \\
\mathrm{IR}=123.82 \mathrm{~g} / \text { day } \\
\text { South } \\
\mathrm{IR}=326.65 \mathrm{~g} / \text { day } \\
(\mathrm{BW}=60 \mathrm{~kg} \text { used for } \\
\text { calculations })\end{array}$ & $\begin{array}{l}\text { North: } 4.7 \times 10^{-4} \\
\text { South: } 8.8 \times 10^{-4}\end{array}$ & $\begin{array}{l}\text { LCR } \\
\text { North: } 0.76 \times 10^{-3} \\
\text { South: } 1.31 \times 10^{-3}\end{array}$ \\
\hline $\begin{array}{l}\text { Southern } \\
\text { Vietnam }\end{array}$ & $\begin{array}{l}\text { Hanh et al. } \\
2011[31]\end{array}$ & $(N=75)$ & $\begin{array}{l}39 \text { rice samples } \\
\text { from } 45 \text { households }\end{array}$ & $\begin{array}{c}\text { Total As } \\
\text { (iAs calculated } \\
\text { from total: } 80 \% \text { ) }\end{array}$ & $\begin{array}{l}\text { Total As: } 0.22 \\
(0.13-0.47)\end{array}$ & $\begin{array}{c}\text { Males } \\
\mathrm{IR}=300 \mathrm{~g} / \text { day } \\
\mathrm{BW}=58 \mathrm{~kg} \\
\text { Females } \\
\mathrm{IR}=250 \mathrm{~g} / \text { day } \\
\mathrm{BW}=50 \mathrm{~kg}\end{array}$ & $\begin{array}{c}\text { Males: } 0.053 \pm 0.018 \\
\text { mg/day } \\
\text { Females: } 0.045 \pm 0.016 \\
\text { mg/day }\end{array}$ & $\begin{array}{l}\text { EDIs were both below the }{ }^{1} \\
\text { BMDL }_{0.5}\end{array}$ \\
\hline
\end{tabular}


Table 2. Cont

\begin{tabular}{|c|c|c|c|c|c|c|c|c|}
\hline $\begin{array}{c}\text { Study } \\
\text { Location }\end{array}$ & $\begin{array}{l}\text { Author(s) } \\
\text { and Year }\end{array}$ & $\begin{array}{l}\text { Age/Group } \\
(N=)\end{array}$ & Source of Arsenic & Type of Arsenic & $\begin{array}{c}\text { Mean (mg/kg) } \pm \text { SD } \\
\text { (Range) }\end{array}$ & $\begin{array}{l}\text { As Exposure Estimation } \\
\text { (IR = Ingestion Rate of Rice) }\end{array}$ & EDI (mg/kg-BW/Day) & Cancer Risk \\
\hline Bengal & $\begin{array}{l}\text { Halder et al. } \\
2013 \text { [32] }\end{array}$ & $(N=157)$ & $\begin{array}{l}157 \text { rice samples } \\
\text { from households }\end{array}$ & $\begin{array}{l}\text { Total As } \\
\text { (fraction of } \\
\text { iAs }=0.92 \text { ) }\end{array}$ & Total As: $0.010-0.64$ & $\begin{array}{c}18-30 \text { years: } \\
200-400 \text { g/day dry weight } \\
\text { 31-50 years: } \\
100-500 \text { g/day } \\
51-65 \text { years: } \\
150-450 \text { g/day } \\
\end{array}$ & - & $\begin{array}{l}\text { When As concentration in } \\
\text { drinking water }<10 \mu \mathrm{g} / \mathrm{L}, 35 \% \\
\text { of participants had total daily } \\
\text { intake of iAs above }{ }^{2} \text { PTDI }\end{array}$ \\
\hline $\begin{array}{l}\text { Hunan } \\
\text { Province of } \\
\text { China }\end{array}$ & $\begin{array}{l}\text { Lei et al. } \\
2013 \text { [33] }\end{array}$ & $\begin{array}{l}\text { Adults \& } \\
\text { children }\end{array}$ & $\begin{array}{l}34 \text { genotypes of rice } \\
\text { grown in } \\
\text { As-contaminated } \\
\text { field (unpolished } \\
\text { rice) }\end{array}$ & Total and iAs & $\begin{array}{c}\text { Total As: } 0.42 \\
(0.31-0.52) \\
\text { iAs: } 0.39(0.26-0.52)\end{array}$ & $\begin{array}{c}\text { Adult } \\
\mathrm{IR}=0.40 \mathrm{~kg} / \text { day (Lin et al. 2004) } \\
\text { Children } \\
\mathrm{IR}=0.29 \mathrm{~kg} / \text { day }\end{array}$ & $\begin{array}{c}\text { Adult } \\
0.10-0.21 \mathrm{mg} \\
\text { Children } \\
0.080-0.15 \mathrm{mg}\end{array}$ & $\begin{array}{c}\text { Most samples exceeded the }{ }^{2} \\
\text { PTDI }\end{array}$ \\
\hline Cambodia & $\begin{array}{l}\text { Phan et al. } \\
2013 \text { [34] }\end{array}$ & Adults & $\begin{array}{l}10 \text { rice samples } \\
\text { from } 3 \text { provinces }\end{array}$ & $\begin{array}{l}\text { iAs assumed to } \\
\text { be } 80 \% \text { of } \\
\text { total As }\end{array}$ & $\begin{array}{c}\text { Kandal } \\
0.20 \pm 0.27(0.0080-0.95) \\
\text { Kratie } \\
0.064 \pm 0.046 \\
(0.0040-0.15) \\
\text { Kampong Cham } \\
0.010 \pm 0.0090 \\
(0.0030-0.025)\end{array}$ & $\begin{array}{l}\text { Rice consumed } 3 \text { times/day } \\
\text { (approx. } 450 \mathrm{~g} / \text { day) } \\
\text { Mean } \mathrm{BW}=52 \mathrm{~kg}\end{array}$ & $\begin{array}{c}\text { Kandal } \\
1.77 \times 10^{-3}(6.80 \times \\
\left.10^{-5}-8.23 \times 10^{-3}\right) \\
\text { Kratie } \\
5.50 \times 10^{-4}(3.70 \times \\
\left.10^{-5}-1.32 \times 10^{-3}\right) \\
\text { Kampong Cham } \\
8.60 \times 10^{-5}(2.80 \times \\
\left.10^{-5}-2.10 \times 10^{-4}\right) \\
\end{array}$ & $\begin{array}{l}\text { Kandal: The upper end of the } \\
\text { EDI range was greater than the } \\
\text { lower limits of }{ }^{1} \mathrm{BMDL}_{0.5}\end{array}$ \\
\hline Hong Kong & $\begin{array}{l}\text { Wong et al. } \\
2013 \text { [35] }\end{array}$ & $\begin{array}{c}20-84 \text { years } \\
(N=5008)\end{array}$ & $\begin{array}{l}600 \text { composite } \\
\text { samples of cooked } \\
\text { white rice }\end{array}$ & iAs & $0.022(0.016-0.026)$ & $\begin{array}{l}\text { Food consumption data taken } \\
\text { from Hong Kong } \\
\text { Population-based Food } \\
\text { Consumption Survey } \\
(2005-2007)\end{array}$ & $\begin{array}{c}2.2 \times 10^{-4} \\
95 \text { th percentile: } \\
3.8 \times 10^{-4}\end{array}$ & ${ }^{3}$ MOE: $9-32$ \\
\hline $\begin{array}{l}\text { Zhejiang, } \\
\text { China }\end{array}$ & $\begin{array}{l}\text { Z. Huang et } \\
\text { al. } 2013 \text { [19] }\end{array}$ & $\begin{array}{l}\text { Adults } \\
>18 \text { years \& } \\
\text { children } \\
7-18 \text { years } \\
(N=9798)\end{array}$ & $\begin{array}{l}248 \text { rice samples } \\
\text { from local markets } \\
\text { in } 2012\end{array}$ & Total As & $0.080\left(<\mathrm{LOD}^{* *}-0.21\right)$ & $\begin{array}{c}\text { (Food consumption survey from } \\
\text { the Zhejiang FDA) } \\
\text { Adults IR }=342.90 \mathrm{~g} / \text { day } \\
\text { Adults BW }=55.9 \mathrm{~kg} \\
\text { Children IR }=258.42 \mathrm{~g} / \text { day } \\
\text { Children } \mathrm{BW}=32.7 \mathrm{~kg}\end{array}$ & $\begin{array}{l}\text { Adults: } 4.9 \times 10^{-4} \\
\text { Children: } 3.4 \times 10^{-4}\end{array}$ & $\begin{array}{l}\text { Health risk index <1.0: no } \\
\text { health risk }\end{array}$ \\
\hline $\begin{array}{l}\text { Southwest } \\
\text { Taiwan }\end{array}$ & $\begin{array}{l}\text { Lamm et al. } \\
2014[36]\end{array}$ & $\begin{array}{c}\text { Adults } \\
>20 \text { years } \\
(N=34,783)\end{array}$ & $\begin{array}{l}\text { Well water from } \\
42 \text { villages }\end{array}$ & Total As & $\begin{array}{l}\text { Means: } 10-818 \mu \mathrm{g} / \mathrm{L} \\
\text { Range: } 10-1752\end{array}$ & $\begin{array}{c}\text { Village well water data from } \\
\text { 1964-1966: } \\
\text { Males: } 3.5 \mathrm{~L} / \text { day } \\
\text { Females: } 2.0 \mathrm{~L} / \text { day } \\
50 \mathrm{~kg} \text { BW for both sexes }\end{array}$ & - & $\begin{array}{l}\text { Crude mortality rates (CMR): } \\
\text { Maximum value of } 2.8 \text { for village } \\
\text { with As median of } 698 \mu \mathrm{g} / \mathrm{L}\end{array}$ \\
\hline Japan & $\begin{array}{l}\text { Oguri et al. } \\
2014[37]\end{array}$ & $(N=1142)$ & $\begin{array}{l}19 \text { food composites } \\
\text { prepared from } \\
159 \text { food items } \\
\text { purchased in } \\
\text { Shizuoka City }\end{array}$ & iAs & Raw rice: 0.18 and 0.095 & $\begin{array}{l}\text { Daily consumption rate of } \\
\text { corresponding food category } \\
\text { (MHLW 2007): } 312.50 \\
\text { g/person/day }\end{array}$ & $\begin{array}{c}\text { Cereals: } \\
0.013 \mathrm{mg} / \text { person/day } \\
\text { (rice \& rice cakes } \\
\text { contributed to } 97 \% \text { of } \\
\text { iAs intake from cereals) }\end{array}$ & $\begin{array}{c}\text { LCR } \\
\text { Skin: } 6.1 \times 10^{-4} \\
\text { Liver and Lung: } 1.2-8.8 \times 10^{-4}\end{array}$ \\
\hline
\end{tabular}


Table 2. Cont

\begin{tabular}{|c|c|c|c|c|c|c|c|c|}
\hline $\begin{array}{l}\text { Study } \\
\text { Location }\end{array}$ & $\begin{array}{l}\text { Author(s) } \\
\text { and Year }\end{array}$ & $\begin{array}{l}\text { Age/Group } \\
(N=)\end{array}$ & Source of Arsenic & Type of Arsenic & $\begin{array}{c}\text { Mean (mg/kg) } \pm S D \\
\text { (Range) }\end{array}$ & $\begin{array}{c}\text { As Exposure Estimation } \\
\text { (IR = Ingestion Rate of Rice) }\end{array}$ & EDI (mg/kg-BW/Day) & Cancer Risk \\
\hline Europe & $\begin{array}{l}\text { Gundert-Remy } \\
\text { et al. } \\
2015[6]\end{array}$ & $\begin{array}{l}\text { Infants to } \\
\geq 75 \text { years }\end{array}$ & "All foods" & iAs & $\begin{array}{c}0.089 \\
(0.084-0.093)\end{array}$ & $\begin{array}{l}\text { European Food Safety Authority } \\
\text { (EFSA) } 2014 \text { survey }\end{array}$ & $\begin{array}{c}\text { Adolescents } \\
\text { (10-17 years) } \\
\text { Exposure from } \\
\text { "all foods": } \\
1.2 \times 10^{-4}-4.8 \times 10^{-4}\end{array}$ & Exposure lower than ${ }^{1} \mathrm{BMDL}_{0.5}$ \\
\hline China & $\begin{array}{l}\text { Jiang et al. } \\
2015[38]\end{array}$ & $\begin{array}{l}2-70 \text { years \& } \\
\text { over } \\
(N=244)\end{array}$ & $\begin{array}{l}\text { Samples } \\
\text { self-cultivated by } \\
\text { inhabitants \& some } \\
\text { from market }\end{array}$ & $\begin{array}{l}\text { Total As (iAs } \\
\text { estimated using } \\
\text { ratio of } \\
\text { iAs/total } \\
\text { As } 26.8 \% \text { ) }\end{array}$ & $\begin{array}{c}\text { Total: } 0.10(0.046-0.25) \\
\text { iAs: } 0.20\end{array}$ & $\begin{array}{l}\text { Data from Survey of the } \\
\text { Nutrition and Health Status } \\
\text { (NHS) of the Chinese People } \\
\text { in } 2002\end{array}$ & $\begin{array}{l}\text { From all foods: } \\
\text { (14-17 years) } \\
\text { Males: } 1.08 \times 10^{-4} \\
\text { Females: } 1.01 \times 10^{-4}\end{array}$ & $\begin{array}{l}\text { LCR from all foods } \\
\quad(14-17 \text { years }) \\
\text { Males: } 1.62 \times 10^{-4} \\
\text { Females: } 1.51 \times 10^{-4}\end{array}$ \\
\hline China & $\begin{array}{l}\text { Y. Huang et } \\
\text { al. } 2015 \text { [9] }\end{array}$ & $2-80$ years & $\begin{array}{l}1653 \text { rice samples } \\
\text { from } 11 \text { provinces }\end{array}$ & iAs & $\begin{array}{c}0.091 \\
(* \mathrm{ND}-0.30)\end{array}$ & $\begin{array}{l}\text { IR and BW from Report on } \\
\text { Nutrition and Health Status of } \\
\text { Chinese Residents (2002) }\end{array}$ & - & $\begin{array}{c}\text { Age } 14-18 \text { years: } \\
\text { Males: }{ }^{3} \mathrm{MOE}=6.28 \\
\text { Females: }{ }^{3} \mathrm{MOE}=7.61\end{array}$ \\
\hline Illinois, USA & $\begin{array}{l}\text { Bulka et al. } \\
2016[39]\end{array}$ & $\begin{array}{c}\text { Males } \\
\geq 15 \text { years } \\
(N=4,936,634)\end{array}$ & Water & Total As & $\begin{array}{c}\text { Mean As tertiles in ppb: } \\
0.33-0.72 \\
0.73-1.60 \\
1.61-16.23 \\
\end{array}$ & $\begin{array}{l}\text { Illinois EPA data on community } \\
\text { water systems (2000-2006) }\end{array}$ & - & $\begin{array}{l}\text { Modeling arsenic as a continuous } \\
\text { variable: } 10 \mathrm{ppb} \text { increase in As } \\
\text { associated with a } 12 \% \text { increase in } \\
\text { SIR for prostate cancer. }\end{array}$ \\
\hline USA & $\begin{array}{l}\text { Shibata et al. } \\
2016[40]\end{array}$ & $\begin{array}{l}\text { Infants } \\
4-24 \mathrm{mo}\end{array}$ & Rice cereal & iAs & $0.091(0.023-0.28)$ & $\begin{array}{l}\text { (U.S. FDA Rice and Rice Product } \\
\text { Sampling (2013) and } \\
\text { Signes-Pastor et al. 2016) } \\
\text { IR }=14.30-51.50 \mathrm{~g} / \text { day } \\
\text { BW }=6.95-11.85 \mathrm{~kg}\end{array}$ & Median: $9.5 \times 10^{-6}$ & $\begin{array}{c}\text { LCR } \\
\text { Median: } 1.4 \times 10^{-5}\end{array}$ \\
\hline $\begin{array}{l}\text { Republic of } \\
\text { Kazakhstan, } \\
\text { Portugal, } \\
\text { and Spain }\end{array}$ & $\begin{array}{l}\text { Tattibayeva } \\
\text { et al. } \\
2016[4]\end{array}$ & - & $\begin{array}{l}95 \text { rice samples } \\
\text { from local markets }\end{array}$ & iAs & $\begin{array}{c}\text { Kazakhstan } \\
\text { (unpolished) } \\
0.36 \pm 0.020 \\
(0.25-0.45) \\
\text { Spain (milled) } \\
0.25 \pm 0.16 \\
(0.15-0.55) \\
\text { Portugal (milled) } \\
0.18 \pm 0.15 \\
(0.10-0.30)\end{array}$ & $\begin{array}{l}\text { Standard adult male BW }=70 \mathrm{~kg} \\
\text { Children } \mathrm{BW}=24 \mathrm{~kg}\end{array}$ & $\begin{array}{c}\text { Average Estimated } \\
\text { Weekly Intake }(\text { EWI }) \\
(\mathrm{mg} / \mathrm{kg}): \\
\text { Kazakh } \\
\text { Adults: } 7.7 \times 10^{-4} \\
\text { Children: } 1.88 \times 10^{-3} \\
\text { Spain } \\
\text { Adults: } 2.9 \times 10^{-4} \\
\text { Children: } 7.0 \times 10^{-4} \\
\text { Portugal } \\
\text { Adults: } 8.4 \times 10^{-4} \\
\text { Children: } 2.04 \times 10^{-3}\end{array}$ & $\begin{array}{l}\text { All EWI values lower than the } \\
\text { lower limit of the }{ }^{1} \mathrm{BMDL}_{0.5}\end{array}$ \\
\hline United States & $\begin{array}{l}\text { U.S. FDA } \\
2016[14]\end{array}$ & $0-50$ years & $\begin{array}{l}481 \text { rice samples } \\
\text { from retail locations } \\
\text { and USA Rice } \\
\text { Federation } \\
\end{array}$ & iAs & $\begin{array}{l}\text { White short-grain } \\
\text { rice:0.079 }\end{array}$ & $\begin{array}{l}\text { Rice intake and each } \\
\text { respondent's BW taken from } \\
\text { NHANES/WWEIA (2009-2010) }\end{array}$ & $\begin{array}{l}\text { Mean per capita iAs } \\
\text { exposure from rice: } \\
\quad 6.6 \times 10^{-7}\end{array}$ & $\begin{array}{l}\text { Median estimated total cancer } \\
\text { (bladder and lung) cases per } \\
\text { million }(90 \% \text { CI) for lifetime: } \\
<1(0,1.7)\end{array}$ \\
\hline
\end{tabular}


Table 2. Cont

\begin{tabular}{|c|c|c|c|c|c|c|c|c|}
\hline $\begin{array}{c}\text { Study } \\
\text { Location }\end{array}$ & $\begin{array}{l}\text { Author(s) } \\
\text { and Year }\end{array}$ & $\begin{array}{l}\text { Age/Group } \\
\qquad(N=)\end{array}$ & Source of Arsenic & Type of Arsenic & $\begin{array}{c}\text { Mean (mg/kg) } \pm S D \\
\text { (Range) }\end{array}$ & $\begin{array}{c}\text { As Exposure Estimation } \\
\text { (IR = Ingestion Rate of Rice) }\end{array}$ & EDI (mg/kg-BW/Day) & Cancer Risk \\
\hline $\begin{array}{l}\text { Southern } \\
\text { China }\end{array}$ & $\begin{array}{l}\text { Zhuang et al. } \\
2016 \text { [41] }\end{array}$ & $\begin{array}{l}\text { Children \& } \\
\text { adults }\end{array}$ & $\begin{array}{c}\text { (Long-grain rice) } \\
\text { Sample A: market } \\
\text { Sample B: } \\
\text { mining area } \\
\text { Sample C: lab } \\
\text { grown }\end{array}$ & Total As & $\begin{array}{l}\text { Sample A: } 0.14 \pm 0.014 \\
\text { Sample B: } 0.17 \pm 0.040 \\
\text { Sample C: } 0.26 \pm 0.077\end{array}$ & $\begin{array}{c}\text { Adults } \\
\mathrm{IR}=389 \mathrm{~g} / \text { day } \\
\mathrm{BW}=60 \mathrm{~kg} \\
\text { Children } \\
\mathrm{IR}=277 \mathrm{~g} / \text { day } \\
\mathrm{BW}=32.5 \mathrm{~kg} \\
\text { (IR data taken from } \\
\text { Wang et al. 2005) }\end{array}$ & $\begin{array}{c}\text { Sample A } \\
\text { Adults: } 5.3 \times 10^{-4} \\
\text { Children: } 7.0 \times 10^{-4} \\
\text { Sample B } \\
\text { Adults: } 7.4 \times 10^{-4} \\
\text { Children: } 1.4 \times 10^{-3} \\
\text { (Based on bioaccessible } \\
\text { concentrations of As) }\end{array}$ & $\begin{array}{c}\text { Target hazard quotient (THQ): } \\
\text { Sample A } \\
\text { Adults: } 1.77 \\
\text { Children: } 2.33 \\
\text { Sample B } \\
\text { Adults: } 2.48 \\
\text { Children: } 4.58 \\
\text { All THQs }>1 \text { : high } \\
\text { non-carcinogenic health risks }\end{array}$ \\
\hline Canada & $\begin{array}{l}\text { Cheasley et } \\
\text { al. } 2017 \text { [42] }\end{array}$ & $\begin{array}{l}(N=34,944) \\
\quad \text { from } \\
10 \text { provinces }\end{array}$ & Rice & $\begin{array}{l}\text { Total As } \\
\text { (iAs: assumed } \\
\text { only } 40 \% \text { of } \\
\text { total As) }\end{array}$ & $\begin{array}{c}\text { Total As: } \\
0.065 \\
(0.036-0.094)\end{array}$ & $\begin{array}{l}\text { Dietary patterns from Health } \\
\text { Canada's Canadian Community } \\
\text { Health Survey } \\
\text { BW }=70 \mathrm{~kg}\end{array}$ & $\begin{array}{c}\text { Urban } \\
\text { Median }=1.8 \times 10^{-3} \\
\mathrm{mg} / \text { day } \\
(0.0-0.075) \\
\text { Rural } \\
\text { Median }=1.3 \times 10^{-3} \\
\mathrm{mg} / \text { day } \\
(0.0-0.053) \\
\end{array}$ & $\begin{array}{l}\text { Used cancer slope factor of } 1.8 \text { : } \\
50-60 \% \text { of EDIs resulted in LCR } \\
\text { values above } 10 \text { per million }\end{array}$ \\
\hline China & $\begin{array}{l}\text { H. B. Li et al. } \\
2017[8]\end{array}$ & Adults & $\begin{array}{l}55 \text { rice samples } \\
\text { from } 15 \text { provinces }\end{array}$ & Total As & $\begin{array}{c}0.13 \\
(0.038-0.34)\end{array}$ & $\begin{array}{l}\text { Assuming IR of } 350 \mathrm{~g} / \text { day and } \\
60 \mathrm{~kg} \text { adult }\end{array}$ & $4.1 \times 10^{-4}-1.5 \times 10^{-3}$ & $\begin{array}{c}\text { Contributing to } 13.7-50.0 \% \text { of }{ }^{1} \\
\text { BMDL }_{0.5}\end{array}$ \\
\hline Bangkok & $\begin{array}{l}\text { Hensawang } \\
\text { et al. } \\
2017[1]\end{array}$ & $\begin{array}{l}\text { 3-65 years \& } \\
\quad \text { over }\end{array}$ & $\begin{array}{c}31 \text { rice samples } \\
\text { from local markets } \\
\text { in } 8 \text { different } \\
\text { clusters of Bangkok } \\
\end{array}$ & Total As & $\begin{array}{l}0.17 \pm 0.0090 \\
(0.084-0.27)\end{array}$ & $\begin{array}{c}\text { Adolescents (9-19 years): } \\
\text { BW }=46.48 \mathrm{~kg} \\
\text { Consumption per capita }= \\
128.58 \mathrm{~g} / \text { day } \\
\end{array}$ & $\begin{array}{l}\text { Adolescents: } \\
3.92 \times 10^{-4}\end{array}$ & $\begin{array}{c}\text { LCR } \\
\text { Adolescents: } 2.15 \times 10^{-8}\end{array}$ \\
\hline Bangladesh & $\begin{array}{l}\text { Islam et al. } \\
2017 \text { [43] }\end{array}$ & Adults & $\begin{array}{l}965 \text { rice samples } \\
\text { from } 73 \\
\text { sub-districts } \\
\text { during } 2014\end{array}$ & iAs & 0.20 & $\begin{array}{c}\text { (Ages 16-19) } \\
\text { Males: } \\
\text { BW }=52 \mathrm{~kg} \\
\mathrm{IR}=482 \mathrm{~g} / \text { day } \\
\text { Females: } \\
\mathrm{BW}=41.4 \mathrm{~kg} \\
\mathrm{IR}=453 \mathrm{~g} / \text { day }\end{array}$ & - & $\begin{array}{c}\text { LCR } \\
\text { (Ages 16-19) } \\
\text { Males: } 2.73 \times 10^{-3} \\
\text { Females: } 3.22 \times 10^{-3}\end{array}$ \\
\hline Poland & $\begin{array}{l}\text { Mania et al. } \\
2017[44]\end{array}$ & $\begin{array}{l}\text { Adults \& } \\
\text { children }\end{array}$ & $\begin{array}{l}62 \text { rice samples \& } \\
\text { rice products } \\
\text { from trade }\end{array}$ & iAs & $\begin{array}{c}\text { White rice } \\
0.030 \\
\text { 90th percentile: } 0.060\end{array}$ & $\begin{array}{c}\text { Data from Central Statistical } \\
\text { Office: } \\
\mathrm{IR}=0.17 \mathrm{~kg} / \mathrm{person} / \text { month } \\
\text { Adult BW }=70 \mathrm{~kg} \\
\text { Children } \mathrm{BW}=20 \mathrm{~kg}\end{array}$ & - & $\begin{array}{l}\mathrm{EDI} \text { (from rice and rice-based } \\
\text { products) } \leq 1 \% \text { of }{ }^{1} \mathrm{BMDL}_{0.5}\end{array}$ \\
\hline Ecuador & $\begin{array}{l}\text { Nunes et al. } \\
2017 \text { [7] }\end{array}$ & $\begin{array}{l}1-59 \text { years } \\
(N=19,932)\end{array}$ & $\begin{array}{l}16 \text { market basket } \\
\text { rice samples \& } \\
26 \text { rice samples } \\
\text { collected directly } \\
\text { from rice paddies }\end{array}$ & $\begin{array}{c}\text { Total As } \\
\text { (iAs estimated } \\
\text { using ratio of } \\
\text { iAs/total As }= \\
0.80 \pm 0.08 \text { ) }\end{array}$ & $\begin{array}{c}\text { Total As } \\
\text { Field rice: } 0.060 \pm 0.052 \\
\text { Market basket rice: } \\
0.070 \pm 0.029\end{array}$ & $\begin{array}{l}\text { IR and BW per age class were } \\
\text { based on } 24 \mathrm{~h} \text { recall study of } \\
\text { Ministry Health and Nutrition }\end{array}$ & $\begin{array}{c}\text { Men }>14 \text { years: } \\
5.4 \times 10^{-5} \\
\text { Women }>14 \text { years: } \\
6.2 \times 10^{-5}\end{array}$ & $\begin{array}{c}\text { LCR } \\
\text { Men >14 years: } 8.5 \times 10^{-5} \\
\text { Women >14 years: } 1.0 \times 10^{-4}\end{array}$ \\
\hline
\end{tabular}


Table 2. Cont.

\begin{tabular}{|c|c|c|c|c|c|c|c|c|}
\hline $\begin{array}{c}\text { Study } \\
\text { Location }\end{array}$ & $\begin{array}{l}\text { Author(s) } \\
\text { and Year }\end{array}$ & $\begin{array}{c}\text { Age/Group } \\
(N=)\end{array}$ & Source of Arsenic & Type of Arsenic & $\begin{array}{c}\text { Mean (mg/kg) } \pm S D \\
\text { (Range) }\end{array}$ & $\begin{array}{c}\text { As Exposure Estimation } \\
\text { (IR = Ingestion Rate of Rice) }\end{array}$ & EDI (mg/kg-BW/Day) & Cancer Risk \\
\hline Malaysia & $\begin{array}{l}\text { Praveena et } \\
\text { al. } 2017 \text { [45] }\end{array}$ & $\begin{array}{l}\text { Adults \& } \\
\text { children }\end{array}$ & $\begin{array}{l}22 \text { varieties of rice } \\
\text { from local } \\
\text { superstores }\end{array}$ & Total As & $0.091 \pm 0.0010$ & $\begin{array}{c}\text { (Values obtained from other } \\
\text { studies) } \\
\text { Adult } \\
\text { IR }=600 \mathrm{~g} / \text { day BW }=62.65 \mathrm{~kg} \\
\text { Children } \\
\text { (data obtained from Chinese } \\
\text { population) } \\
\text { IR }=198.4 \mathrm{~g} / \mathrm{day} \\
\mathrm{BW}=19.5 \mathrm{~kg}\end{array}$ & - & $\begin{array}{c}\text { LCR } \\
\text { Adult: } 4.9 \times 10^{-3} \\
\text { Children: } 3.2 \times 10^{-3}\end{array}$ \\
\hline Pakistan & $\begin{array}{l}\text { Rasheed et } \\
\text { al. } 2017 \text { [46] }\end{array}$ & $\begin{array}{l}(N=398) \\
66 \text { children } \\
<16 \text { years \& } \\
332 \text { adults } \\
\geq 16 \text { years }\end{array}$ & Rice & Total As & $0.082 \pm 0.054$ & $\begin{array}{c}\text { (Data from Rasheed et al. 2016) } \\
\text { Ages } 6-16 \\
\text { IR }=272 \mathrm{~g} / \text { day } \\
\mathrm{BW}=26 \mathrm{~kg} \\
\text { Adults }>16 \text { years } \\
\text { Male } \mathrm{IR}=576 \mathrm{~g} / \text { day } \\
\text { Male } \mathrm{BW}=68 \mathrm{~kg} \\
\text { Female } \mathrm{IR}=463 \mathrm{~g} / \text { day } \\
\text { Female } \mathrm{BW}=55 \mathrm{~kg}\end{array}$ & - & $\begin{array}{c}\text { Age 6-16 } \\
1.4 \times 10^{-3} \\
\text { Age } 16-67 \\
8.0 \times 10^{-4} \\
\text { Used Age Dependent } \\
\text { Adjustment Factors (ADAF) }\end{array}$ \\
\hline $\begin{array}{l}\text { Northwestern } \\
\text { Thailand }\end{array}$ & $\begin{array}{l}\text { Chanpiwat } \\
\text { et al. } \\
2018 \text { [47] }\end{array}$ & Adults & $\begin{array}{l}59 \text { locally grown } \\
\text { rice samples }\end{array}$ & $\begin{array}{l}\text { iAs estimated } \\
\text { assuming } \\
63.2-63.5 \% \text { of } \\
\text { total As is iAs }\end{array}$ & $\begin{array}{l}\text { iAs: } 0.20 \pm 0.0070 \\
\quad(0.12-0.29)\end{array}$ & $\begin{array}{c}\mathrm{IR}=84.98 \mathrm{~g} / \text { day } \\
\text { Life expectancy }=75 \text { years } \\
\text { Average } \mathrm{BW}=63.15 \mathrm{~kg}\end{array}$ & $\begin{array}{c}2.0 \times 10^{-4} \pm 6.6 \times \\
10^{-5} \\
\left(1.1 \times 10^{-4}-3.5 \times 10^{-4}\right) \\
\text { Bioaccessible As }\end{array}$ & $\begin{array}{c}\mathrm{LCR} \\
4.0 \times 10^{-4} \pm 9.0 \times 10^{-5} \\
\left(2.4 \times 10^{--4}-6.6 \times 10^{-4}\right)\end{array}$ \\
\hline
\end{tabular}

${ }^{1} \mathrm{BMDL}_{0.5}$ : iAs lower limit on the benchmark dose for a $0.5 \%$ increased incidence of lung cancer $=3.0 \times 10^{-3} \mathrm{mg} / \mathrm{kg}$-BW-day [10]; ${ }^{2} \mathrm{PTDI}$ : previous provisional tolerable daily intake recommended by the WHO: $2.1 \times 10^{-3} \mathrm{mg} / \mathrm{kg}$-bw $/$ day [21]; ${ }^{3} \mathrm{MOE}$ : ratio of $\mathrm{BMDL} 0.5$ to iAs dietary exposure. MOE $<100$ means significant risk of carcinogenic effects [9]; ${ }^{*} \mathrm{ND}=$ not detectable; ** LOD $=$ limit of detection. 
Figure 2 compares studies that directly measured iAs concentrations in rice. The Chinese maximum contaminant level (MCL) for iAs in polished rice is $0.2 \mathrm{mg} / \mathrm{kg}$ [48] and the graph displays that many studies measured iAs concentrations well below the MCL, which may or may not be health protective depending on corresponding rice consumption rates.

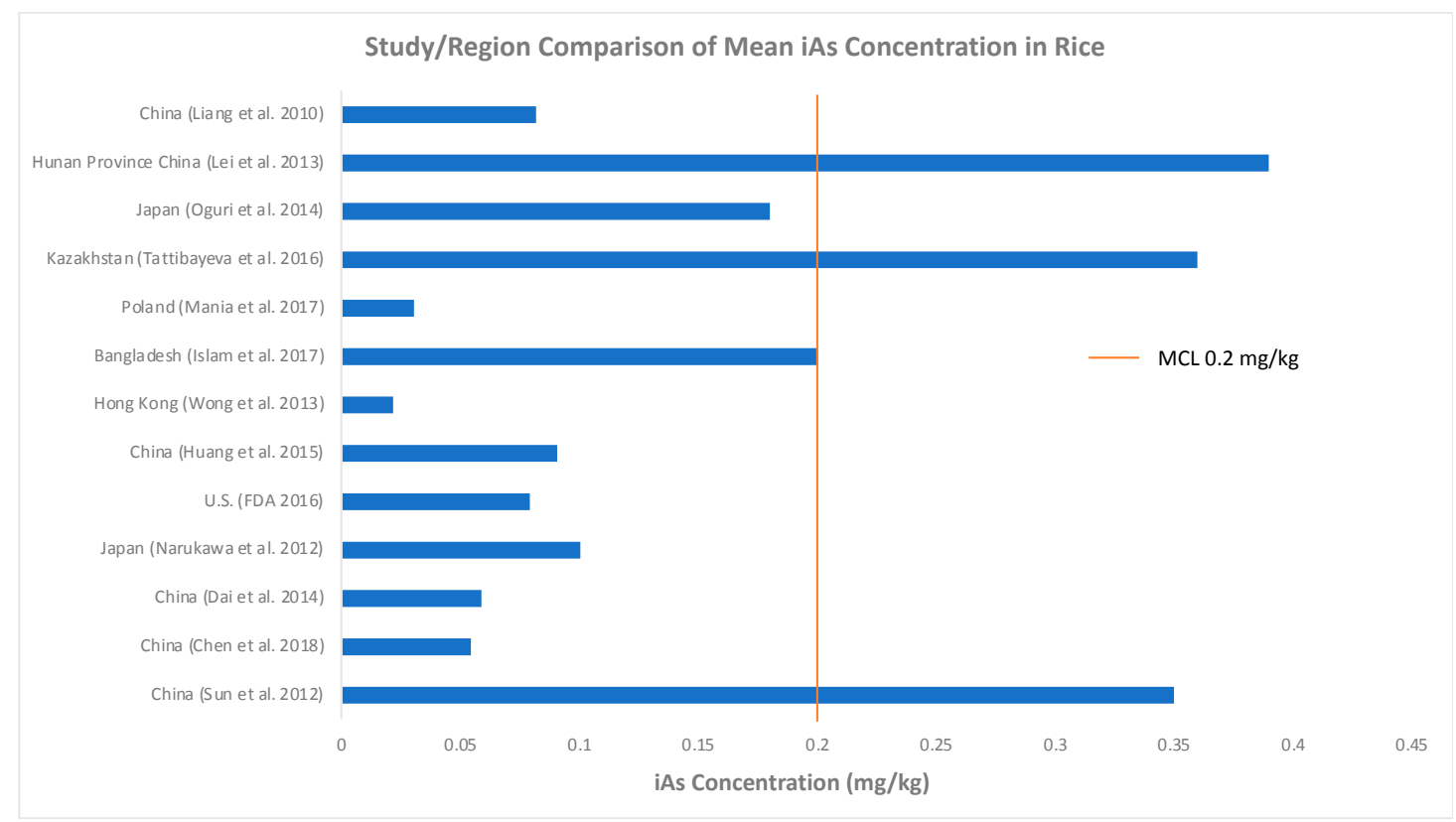

Figure 2. Comparison of iAs concentrations in rice in different studies and regions.

\section{2. iAs Concentrations in Sampled Rice}

The iAs concentrations measured in the Kunming samples ranged from $0.045-0.076 \mathrm{mg} / \mathrm{kg}$ and are below the Chinese MCL. Of the two samples from the same location (one sample blinded to the commercial laboratory), total arsenic concentrations were $0.080 \mathrm{mg} / \mathrm{kg}$ for both samples and iAs concentrations were $0.054 \mathrm{mg} / \mathrm{kg}$ and $0.050 \mathrm{mg} / \mathrm{kg}$. This provided assurance of the reliability of the lab measurement procedures. Interestingly, the sample from the high-end market selling organic food had the second highest level of iAs (0.067), which demonstrates that price and quality of products may not be a factor that reduces arsenic contamination in food products. These seven measurements averaged to be $0.058 \mathrm{mg} / \mathrm{kg}$ (markedly the same mean value for Southern China measured by Chen et al. [20]). We applied this value for our current risk assessment for both males and females.

\subsection{Dietary Rice Consumption}

Among the study participants, males consumed on average $409.9 \mathrm{~g} /$ day of rice, $22 \%$ higher than that of females $(336.6 \mathrm{~g} /$ day). $t$-test results $(p<0.001)$ indicated that males and females differed significantly in their average daily consumption rate of rice. Due to uncertainties in assessing rice content in popular rice products such as rice cakes and rice snacks, calculated consumption rates did not take into account these products. Thus, we may have underestimated the $\mathrm{g} /$ day of rice consumption in the study participants.

\subsection{Estimated Daily Intake of $i$ As and Cancer Risk}

Table 3 displays the values used to calculate the EDI and LCR for adolescents in this study. The mean BW for males was $67.5 \mathrm{~kg}$ (range 44.0-115.9 kg; SD $14.4 \mathrm{~kg}$ ) and for females it was $55.5 \mathrm{~kg}$ (range 37.5-99.4 kg; SD $10.6 \mathrm{~kg}$ ). $t$-test results $(p<0.001)$ indicating that males and females differed significantly in their body weights. The EDI for males was estimated to be $3.52 \times 10^{-4} \mathrm{mg} / \mathrm{kg}-\mathrm{BW} /$ day 
(max. $5.00 \times 10^{-4} \mathrm{mg} / \mathrm{kg}$-BW/day) and for females was estimated to be $3.52 \times 10^{-4} \mathrm{mg} / \mathrm{kg}$-BW $/$ day (max. $5.35 \times 10^{-4} \mathrm{mg} / \mathrm{kg}-\mathrm{BW} /$ day).

Table 3. EDI and LCR Calculations.

\begin{tabular}{cccccc}
\hline \multirow{2}{*}{ Males } & $\begin{array}{c}\text { Mean BW (kg) } \\
\pm \text { SD (Range) }\end{array}$ & $\begin{array}{c}\text { ADC (g/Day) } \\
\pm \text { SD (Range) }\end{array}$ & $\begin{array}{c}\text { AC of iAs (mg/kg) } \\
\pm \text { SD (Range) }\end{array}$ & $\begin{array}{c}\text { EDI (mg/kg-BW/Day) } \\
\text { (Range) }\end{array}$ & LCR (Range) \\
\hline \multirow{2}{*}{ Females } & $67.5 \pm 14.4$ & $409.9 \pm 210.7$ & $0.058 \pm 0.012$ & $3.52 \times 10^{-4}$ & $5.28 \times 10^{-4}$ \\
& $(44.0-115.9)$ & $(0.0-1000.0)$ & $(0.045-0.076)$ & $\left(0-5.00 \times 10^{-4}\right)$ & $\left(0-7.51 \times 10^{-4}\right)$ \\
\hline
\end{tabular}

The calculated average LCR for both males and females using the cancer slope factor of $1.5(\mathrm{mg} / \mathrm{kg}) /$ day was $5.28 \times 10^{-4}\left(\max .7 .51 \times 10^{-4}\right)$, which is 5 times above the upper limit of the U.S. EPA LCR of $1.0 \times 10^{-4}$. Due to differences in body weight and ADC, the calculated maximum LCR for females was $8.02 \times 10^{-4}$, which is eight times above the upper limit of the LCR.

\section{Discussion}

\subsection{Risk among Kunming Adolescents}

The mean iAs concentration in this study's rice samples was $0.058 \mathrm{mg} / \mathrm{kg}$ and the lifetime cancer risk for both males and females in this study cohort was estimated to be $5.28 \times 10^{-4}$. Even though measured iAs concentrations in the sampled rice in this study were below the Chinese MCL, the high consumption rates of rice in this cohort are driving LCR values beyond the LCR acceptable upper limit. Our cohort's LCR was over three times greater than the LCR of adolescents in Jiang et al., which used consumption data from the Survey of the Nutrition and Health Status (NHS) of the Chinese People in 2002 to estimate iAs exposure from all foods measured, not just rice. In that study, the authors did not directly measure iAs, but used conversion factor of $20.3 \%$ to estimate iAs from total arsenic [38]. Awata et al. used the NHANES (2001-2012) data which was the first NHANES cycle to oversample Asians in the U.S. and found that Chinese adolescents (19-21 years old) had iAs dietary intakes of $8 \times 10^{-5} \mathrm{mg} / \mathrm{kg}-\mathrm{BW} /$ day, which is 4.4 times lower than that of our study cohort [49]. This may be due to different eating habits between Chinese and Chinese-American adolescents.

\subsection{Arsenic in Rice and Cancer Risk}

The current Chinese MCL of $0.2 \mathrm{mg} / \mathrm{kg}$, reflective of the maximum level set by the Codex Alimentarius Commission, was raised from the 2005 Chinese MCL of $0.15 \mathrm{mg} / \mathrm{kg}$ [48]. Given the high consumption rates of rice in China, increasing the MCL may inadvertently increase the risk of iAs-related cancer in this country. Based on the adolescent's rice consumption data collected for this study, if the cohort is being exposed at MCL of $0.2 \mathrm{mg} / \mathrm{kg}$, the EDI and LCR estimations would be $12.1 \times 10^{-4}$ and $18.2 \times 10^{-4} \mathrm{mg} / \mathrm{kg}-\mathrm{BW} /$ day for both males and females, respectively. The estimated LCR would be 18 times higher than the upper limit of the U.S. EPA LCR of $1.0 \times 10^{-4}$.

Our cancer risk estimations accounted for "lifetime" exposure to iAs from rice. Although humans may not be consuming rice during infancy, many infant foods are rice-based and contain As. For example, infant rice cereal products sold in the U.S. were shown to have As levels ranging from 0.050 to $0.72 \mathrm{mg} / \mathrm{kg}$, with the lower end of this range being similar to the mean level of iAs found in this study's rice samples [40]. Importantly, chronic exposure to iAs during early life years may greatly increase one's lifetime cancer risk [46]. Rasheed et al. took this increased risk into account by using age dependent adjustment factors (ADAFs). The study estimated cancer risk from arsenic in rice to be $1.4 \times 10^{-3}$ among children (ages $6-16$ ) and $8.0 \times 10^{-4}$ among adults (ages 16-67). By using ADAFs, $0-2$ year olds acquire 10 times the cancer risk and 2-16 year olds acquire 3 times the cancer risk compared to adults [46]. If we account for an ADAF in our LCR calculation, the average LCR for 
the 15 and 16 year olds in this cohort will be $15.84 \times 10^{-4}$, which is approximately 16 times higher than the U.S. EPA LCR limit.

\subsection{Study Strengths and Limitations}

As with most of the previously published studies, the limitations of this study include the cross-sectional nature of the study and the lack of long-term exposure history, which restrain our confidence in any causal predictions of arsenic-attributable health risks. Another limitation is the small sample size in both the rice samples collected and number of study participants, which can decrease study power and generalizability to the adolescents living in other parts of China. Additionally, although LCR values across studies could not be confidently compared given that exposure estimation methods are not uniform, the graph in Figure 1 is valuable as it shows overall differences in cancer risks reported by various studies in the published literature.

The bioavailability of arsenic after ingestion of white rice has been measured to be about $40 \%$ from animal models and between $55-71 \%$ from in vitro studies [8]. Despite this large variation in arsenic bioavailability, it is another factor that should be considered. Furthermore, it is best to base cancer risk calculations on the maximum potential exposure level because it sets the maximum potential risk or "worst case scenario" [1]. We did not assess biomarkers of exposure to As such as in participants' blood and urine (indicator of short-term exposure) and in hair, skin, or nails (indicator of long-term exposure) [31], which may generate much more accurate exposure and dose-response levels.

As exposure rates from rice can vary day-to-day due to inconsistent diet consumptions, different As concentrations and speciation in rice, rice from different geographical sources, and different rinsing and cooking methods [15]. Cooking rice with iAs contaminated water using methods that result in all cooking water being absorbed into the rice may increase iAs exposure from rice [12]. Although Kunming rice cooking techniques were not analyzed in this study, most Chinese residents cook their rice by simmering with the lid closed until all the water is absorbed. Thorough rinsing and cooking rice in high volumes of water and draining excess water have been found to significantly reduce iAs content in cooked rice [46].

Despite these limitations, this study has distinctive strengths. As displayed in Tables 1 and 2, many studies use a fraction of iAs from the total arsenic measured to estimate iAs concentrations in rice. The fractions vary considerably, revealing the variability of arsenic speciation in rice and the uncertainty of iAs levels in rice unless directly measured. This study utilized individual-level data which allows for more accurate exposure estimations and directly measured iAs concentrations in rice. Lastly, market rice samples and individual level data are all from a specific region and this is necessary in arsenic exposure estimation due to the many variable factors discussed earlier.

\subsection{Limitations of Oral Slope Factor}

The current U.S. EPA iAs oral slope factor of $1.5(\mathrm{mg} / \mathrm{kg}) /$ day was last revised in 1995 and is based on old studies $[50,51]$ using skin cancer as the only outcome analyzed [5]. The Tseng studies were cross-sectional and analyzed 40,000 Taiwanese exposed to arsenic in drinking water. The researchers found significant excess skin cancer prevalence by comparison to 7500 residents of Taiwan and Matsu who consumed nearly arsenic-free water. Cancer risk estimates were based on water exposure and no data is given on arsenic exposure from food. Additionally, the slope factor was developed using a low-dose extrapolation procedure from higher doses. Reasoning for the use of such old studies are the very large sample size $(N>40,000)$ and the use of a control group that had no evidence of skin lesions or black foot disease for a sensitive endpoint. This is considered superior for developing a reference dose for oral exposure (RfD). Moreover, this data from Taiwan is of value because it removes potential exposure misclassification due to villagers residing in the same location most of their lives, therefore reflecting long-term exposure to regional As levels [52]. Lastly by not taking into consideration internal cancers such as lung and bladder, the oral slope factor for iAs and subsequent cancer calculations may 
be highly underestimated [5]. Newer oral slope factor proposals are about 17 times greater than the current factor [52].

\subsection{Public Health Implications}

Because China is the highest rice producing and consuming country in the world and accounts for a third of the global supply of rice [9], As-monitoring and enhancing irrigation procedures should be top agricultural priorities in As-affected regions [18]. Future studies should assess changing As levels in agricultural soils over time within the same regions. In particular, the Hunan Province should be a region of focus because Hunan yields roughly 18 million tons of rice annually and includes about 7000 different mines with the potential for environmental contamination [33]. Other important regions include rice growing regions such as Yunnan, Jiangsu, Heibei, Guangdong, Heilongiiang, etc. [18]. There is also a need for increased clinical biomonitoring of high-risk populations in China and more public awareness of the long-term health impacts of iAs and potential exposure from rice. Furthermore, people should be educated about diversifying grains consumed in their diet, washing rice thoroughly before cooking, and cooking rice in higher quantities of water and draining the excess.

\section{Conclusions}

The results of our literature review and analysis reveal that exposure rates and lifetime cancer risk estimations can vary widely between countries, between populations within countries, and between age groups within specific populations. Our study results also suggest that there should be ongoing risk assessments in specific regions, especially in Southern China where residents may be at risk for high exposure levels. Younger populations may be particularly at risk for long-term health effects of chronic iAs exposure from rice because they are highly exposed during earlier years of life and while they are still developing and they have the potential for long-term exposure. In addition, future studies should assess biomarkers of exposure to As such as in participants' blood, urine, hair, skin, or nails [31]. There are concerns that setting a "too low" MCL could potentially harm the rice industry, but policies need to change if these levels are putting populations at high risk [3]. In perspective, this study cohort's lifetime cancer risk of $5.28 \times 10^{-4}$ is 5.28 times higher than the U.S. EPA upper limit of 1 in 10,000, with the upper limit being 100 times higher than the universally accepted cancer risk for an environmental carcinogen of one case/one million people [15].

Author Contributions: Study design: J.H., E.S., B.E. and N.L. Data collection: J.H., E.S., Y.L. and N.L. Data analysis: N.L. and J.H. Manuscript writing: N.L. and J.H. Manuscript editing: all authors.

Funding: This study was funded by Pamela and Kenneth Fong Graduate Student Fellowship, Center for Chinese Studies at UC Berkeley. Jenna Hua was supported by the Ruth L. Kirschstein National Research Service Award for Individual Predoctoral Fellows (Parent F31), National Cancer Institute (NCI), National Institute of Health (NIH), and is currently supported by the Postdoctoral Fellowship in Cardiovascular Disease Prevention (T32), National Heart, Lung and Blood Institute (NHLBI), NIH.

Acknowledgments: The authors would like to thank Craig Steinmaus from UC Berkeley School of Public Health and Shohreh F. Farzan from USC Keck School of Medicine for their insights and support. We also acknowledge Sue Eom for her support with cleaning the cohort data.

Conflicts of Interest: The authors declare no conflict of interest.

\section{Abbreviations}

(ADAF) Age Dependent Adjustment Factor

(As) Arsenic

(ADC) Average Daily Consumption Rate of Rice

$\left(\mathrm{BMDL}_{0.5}\right) \quad$ Benchmark Dose for a $0.5 \%$ Increase in Lung Cancer Incidence

(BW) Body Weight Body Weight Body Weight Body Weight

(EDI) Estimated Daily Intake of Inorganic Arsenic

(FAO) Food and Agriculture Organization of the United Nations 


$\begin{array}{ll}\text { (IR) } & \text { Ingestion Rate of Rice } \\ \text { (iAs) } & \text { Inorganic Arsenic } \\ \text { (AC) } & \text { Inorganic Arsenic Concentration } \\ \text { (SF) } & \text { Inorganic Arsenic Oral Slope Factor } \\ \text { (IARC) } & \text { International Agency for Research on Cancer } \\ \text { (LCR) } & \text { Lifetime Cancer Risk } \\ \text { (LOD) } & \text { Limit of Detection } \\ \text { (MOE) } & \text { Margin of Exposure } \\ \text { (MCL) } & \text { Maximum Contaminant Level in China } \\ \text { (ND) } & \text { Not Detectable } \\ \text { (PTDI) } & \text { Previous Provisional Tolerable Daily Intake } \\ \text { (MOE) } & \text { Ratio of BMDL } 0.5 \text { to iAs Dietary Exposure } \\ \text { (U.S. EPA) } & \text { United States Environmental Protection Agency } \\ \text { (NHANES) } & \text { United States National Health and Nutrition Examination Survey } \\ \text { (WHO) } & \text { World Health Organization }\end{array}$

\section{References}

1. Hensawang, S.; Chanpiwat, P. Health impact assessment of arsenic and cadmium intake via rice consumption in Bangkok, Thailand. Environ. Monit. Assess. 2017, 189, 599. [CrossRef] [PubMed]

2. Signes-Pastor, A.J.; Carey, M.; Meharg, A.A. Inorganic arsenic in rice-based products for infants and young children. Food Chem. 2016, 191, 128-134. [CrossRef] [PubMed]

3. Schmidt, C.W. In search of "just right": The challenge of regulating arsenic in rice. Environ. Health Perspect. 2015, 123, A16-A19. [CrossRef] [PubMed]

4. Tattibayeva, D.; Nebot, C.; Miranda, J.M.; Cepeda, A.; Mateyev, E.; Erkebaev, M.; Franco, C.M. A study on toxic and essential elements in rice from the Republic of Kazakhstan: Comparing the level of contamination in rice from the European Community. Environ. Geochem. Health 2016, 38, 85-98. [CrossRef] [PubMed]

5. United States Environmental Protection Agency. Arsenic, Inorganic; CASRN 7440-38-2. 1988. Available online: http:/ / www.webcitation.org/71CfwBK0x (accessed on 26 July 2018).

6. Gundert-Remy, U.; Damm, G.; Foth, H.; Freyberger, A.; Gebel, T.; Golka, K.; Röhl, C.; Schupp, T.; Wollin, K.M.; Hengstler, J.G. High exposure to inorganic arsenic by food: The need for risk reduction. Arch. Toxicol. 2015, 89, 2219-2227. [CrossRef] [PubMed]

7. Nunes, L.M.; Otero, X. Quantification of health risks in Ecuadorian population due to dietary ingestion of arsenic in rice. Environ. Sci. Pollut. Res. Int. 2017, 24, 27457-27468. [CrossRef] [PubMed]

8. Li, H.B.; Li, J.; Zhao, D.; Li, C.; Wang, X.J.; Sun, H.J.; Juhasz, A.L.; Ma, L.Q. Arsenic Relative Bioavailability in Rice Using a Mouse Arsenic Urinary Excretion Bioassay and Its Application to Assess Human Health Risk. Environ. Sci. Technol. 2017, 51, 4689-4696. [CrossRef] [PubMed]

9. Huang, Y.; Wang, M.; Mao, X.; Qian, Y.; Chen, T.; Zhang, Y. Concentrations of Inorganic Arsenic in Milled Rice from China and Associated Dietary Exposure Assessment. J. Agric. Food Chem. 2015, 63, 10838-10845. [CrossRef] [PubMed]

10. Evaluation of Certain Contaminants in Food. 2011. Available online: http:/ / www.webcitation.org/71rmFlJ14 (accessed on 22 August 2018).

11. Li, G.; Sun, G.X.; Williams, P.N.; Nunes, L.; Zhu, Y.G. Inorganic arsenic in Chinese food and its cancer risk. Environ. Int. 2011, 37, 1219-1225. [CrossRef] [PubMed]

12. Cubadda, F.; Jackson, B.P.; Cottingham, K.L.; Van Horne, Y.O.; Kurzius-Spencer, M. Human exposure to dietary inorganic arsenic and other arsenic species: State of knowledge, gaps and uncertainties. Sci. Total Environ. 2017, 579, 1228-1239. [CrossRef] [PubMed]

13. Liu, C.P.; Luo, C.L.; Gao, Y.; Li, F.B.; Lin, L.W.; Wu, C.A.; Li, X.D. Arsenic contamination and potential health risk implications at an abandoned tungsten mine, southern China. Environ. Pollut. 2010, 158, 820-826. [CrossRef] [PubMed]

14. Food and Drug Administration. Arsenic in Rice and Rice Products Risk Assessment Report. Available online: http:/ / www.webcitation.org/71ChM76sO (accessed on 22 August 2018). 
15. Lai, P.Y.; Cottingham, K.L.; Steinmaus, C.; Karagas, M.R.; Miller, M.D. Arsenic and Rice: Translating Research to Address Health Care Providers' Needs. J. Pediatr. 2015, 167, 797-803. [CrossRef] [PubMed]

16. Petry, M.; Liting, B. National Food Safety Standard. Available online: http://www.webcitation.org/ 71nbirkdu (accessed on 22 August 2018).

17. Ma, L.; Wang, L.; Jia, Y.; Yang, Z. Arsenic speciation in locally grown rice grains from Hunan Province, China: Spatial distribution and potential health risk. Sci. Total Environ. 2016, 557-558, 438-444. [CrossRef] [PubMed]

18. Fang, Y.; Sun, X.; Yang, W.; Ma, N.; Xin, Z.; Fu, J.; Liu, X.; Liu, M.; Mariga, A.M.; Zhu, X.; et al. Concentrations and health risks of lead, cadmium, arsenic, and mercury in rice and edible mushrooms in China. Food Chem. 2014, 147, 147-151. [CrossRef] [PubMed]

19. Huang, Z.; Pan, X.D.; Wu, P.G.; Han, J.L.; Chen, Q. Health risk assessment of heavy metals in rice to the population in Zhejiang, China. PLoS ONE 2013, 8, e75007. [CrossRef] [PubMed]

20. Chen, H.; Tang, Z.; Wang, P.; Zhao, F.J. Geographical variations of cadmium and arsenic concentrations and arsenic speciation in Chinese rice. Environ. Pollut. 2018, 238, 482-490. [CrossRef] [PubMed]

21. Ahmed, M.K.; Shaheen, N.; Islam, M.S.; Habibullah-Al-Mamun, M.; Islam, S.; Islam, M.M.; Kundu, G.K.; Bhattacharjee, L. A comprehensive assessment of arsenic in commonly consumed foodstuffs to evaluate the potential health risk in Bangladesh. Sci. Total Environ. 2016, 544, 125-133. [CrossRef] [PubMed]

22. Dakeishi, M.; Murata, K.; Tamura, A.; Iwata, T. Relation between benchmark dose and no-observed-adverse-effect level in clinical research: Effects of daily alcohol intake on blood pressure in Japanese salesmen. Risk Anal. 2006, 26, 115-123. [CrossRef] [PubMed]

23. He, Y.; Zheng, Y. Assessment of in vivo bioaccessibility of arsenic in dietary rice by a mass balance approach. Sci. Total Environ. 2010, 408, 1430-1436. [CrossRef] [PubMed]

24. Narukawa, T.; Hioki, A.; Chiba, K. Speciation and monitoring test for inorganic arsenic in white rice flour. J. Agric. Food Chem. 2012, 60, 1122-1127. [CrossRef] [PubMed]

25. Norton, G.J.; Pinson, S.R.; Alexander, J.; McKay, S.; Hansen, H.; Duan, G.L.; Rafiqul Islam, M.; Islam, S.; Stroud, J.L.; Zhao, F.J.; et al. Variation in grain arsenic assessed in a diverse panel of rice (Oryza sativa) grown in multiple sites. New Phytol. 2012, 193, 650-664. [CrossRef] [PubMed]

26. Sun, G.X.; Van de Wiele, T.; Alava, P.; Tack, F.; Du Laing, G. Arsenic in cooked rice: Effect of chemical, enzymatic and microbial processes on bioaccessibility and speciation in the human gastrointestinal tract. Environ. Pollut. 2012, 162, 241-246. [CrossRef] [PubMed]

27. Dai, S.; Yang, H.; Mao, X.; Qiu, J.; Liu, Q.; Wang, F.; Wang, M. Evaluation of arsenate content of rice and rice bran purchased from local markets in the People's Republic of China. J. Food Prot. 2014, 77, 665-669. [CrossRef] [PubMed]

28. Liang, F.; Li, Y.; Zhang, G.; Tan, M.; Lin, J.; Liu, W.; Lu, W. Total and speciated arsenic levels in rice from China. Food Addit. Contam. Part A Chem. Anal. Control Expo. Risk Assess. 2010, 27, 810-816. [CrossRef] [PubMed]

29. Mondal, D.; Banerjee, M.; Kundu, M.; Banerjee, N.; Bhattacharya, U.; Giri, A.K.; Ganguli, B.; Sen Roy, S.; Polya, D.A. Comparison of drinking water, raw rice and cooking of rice as arsenic exposure routes in three contrasting areas of West Bengal, India. Environ. Geochem. Health 2010, 32, 463-477. [CrossRef] [PubMed]

30. Adomako, E.E.; Williams, P.N.; Deacon, C.; Meharg, A.A. Inorganic arsenic and trace elements in Ghanaian grain staples. Environ. Pollut. 2011, 159, 2435-2442. [CrossRef] [PubMed]

31. Hanh, H.T.; Kim, K.W.; Bang, S.; Hoa, N.M. Community Exposure to Arsenic in the Mekong River Delta, Southern Vietnam. J. Environ. Monit. 2011, 13, 2025-2232. [CrossRef] [PubMed]

32. Halder, D.; Bhowmick, S.; Biswas, A.; Chatterjee, D.; Nriagu, J.; Guha Mazumder, D.N.; Šlejkovec, Z.; Jacks, G.; Bhattacharya, P. Risk of Arsenic Exposure from Drinking Water and Dietary Components: Implications for Risk Management in Rural Bengal. Environ. Sci. Technol. 2013, 47, 1120-1127. [CrossRef] [PubMed]

33. Lei, M.; Tie, B.; Zeng, M.; Qing, P.; Song, Z.; Williams, P.N.; Huang, Y. An arsenic-contaminated field trial to assess the uptake and translocation of arsenic by genotypes of rice. Environ. Geochem. Health 2013, 35, 379-390. [CrossRef] [PubMed]

34. Phan, K.; Sthiannopkao, S.; Heng, S.; Phan, S.; Huoy, L.; Wong, M.H.; Kim, K.W. Arsenic Contamination in the Food Chain and Its Risk Assessment of Populations Residing in the Mekong River Basin of Cambodia. J. Hazard. Mater. 2013, 262, 1064-1071. [CrossRef] [PubMed] 
35. Wong, W.W.; Chung, S.W.; Chan, B.T.; Ho, Y.Y.; Xiao, Y. Dietary exposure to inorganic arsenic of the Hong Kong population: Results of the first Hong Kong total diet study. Food Chem. Toxicol. 2013, 51, 379-385. [CrossRef] [PubMed]

36. Lamm, S.H.; Robbins, S.; Chen, R.; Lu, J.; Goodrich, B.; Feinleib, M. Discontinuity in the cancer slope factor as it passes from high to low exposure levels-Arsenic in the BFD-endemic area. Toxicology 2014, 326, $25-35$. [CrossRef] [PubMed]

37. Oguri, T.; Yoshinaga, J.; Tao, H.; Nakazato, T. Inorganic arsenic in the Japanese diet: Daily intake and source. Arch. Environ. Contam. Toxicol. 2014, 66, 100-112. [CrossRef] [PubMed]

38. Jiang, Y.; Zeng, X.; Fan, X.; Chao, S.; Zhu, M.; Cao, H. Levels of arsenic pollution in daily foodstuffs and soils and its associated human health risk in a town in Jiangsu Province, China. Ecotoxicol. Environ. Saf. 2015, 122, 198-204. [CrossRef] [PubMed]

39. Bulka, C.M.; Jones, R.M.; Turyk, M.E.; Stayner, L.T.; Argos, M. Arsenic in drinking water and prostate cancer in Illinois counties: An ecologic study. Environ. Res. 2016, 148, 450-456. [CrossRef] [PubMed]

40. Shibata, T.; Meng, C.; Umoren, J.; West, H. Risk Assessment of Arsenic in Rice Cereal and Other Dietary Sources for Infants and Toddlers in the U.S. Int. J. Environ. Res. Public Health 2016, 13, 361. [CrossRef] [PubMed]

41. Zhuang, P.; Zhang, C.; Li, Y.; Zou, B.; Mo, H.; Wu, K.; Wu, J.; Li, Z. Assessment of influences of cooking on cadmium and arsenic bioaccessibility in rice, using an in vitro physiologically-based extraction test. Food Chem. 2016, 213, 206-214. [CrossRef] [PubMed]

42. Cheasley, R.; Keller, C.P.; Setton, E. Lifetime excess cancer risk due to carcinogens in food and beverages: Urban versus rural differences in Canada. Can. J. Public Health 2017, 108, e288-e295. [CrossRef] [PubMed]

43. Islam, S.; Rahman, M.M.; Islam, M.R.; Naidu, R. Geographical variation and age-related dietary exposure to arsenic in rice from Bangladesh. Sci. Total Environ. 2017, 601-602, 122-131. [CrossRef] [PubMed]

44. Mania, M.; Rebeniak, M.; Szynal, T.; Starska, K.; Wojciechowska-Mazurek, M.; Postupolski, J. Exposure assessment of the population in Poland to the toxic effects of arsenic compounds present in rice and rice based products. Roczniki Państwowego Zakładu Higieny 2017, 68, 339-346. [PubMed]

45. Praveena, S.M.; Omar, N.A. Heavy metal exposure from cooked rice grain ingestion and its potential health risks to humans from total and bioavailable forms analysis. Food Chem. 2017, 235, 203-211. [CrossRef] [PubMed]

46. Rasheed, H.; Slack, R.; Kay, P.; Gong, Y.Y. Refinement of arsenic attributable health risks in rural Pakistan using population specific dietary intake values. Environ. Int. 2017, 99, 331-342. [CrossRef] [PubMed]

47. Chanpiwat, P.; Hensawang, S.; Suwatvitayakorn, P.; Ponsin, M. Risk Assessment of Bioaccessible Arsenic and Cadmium Exposure through Rice Consumption in Local Residents of Themae Tao Sub-District, Northwestern Thailand. Environ. Geochem. Health 2018, 1-14. [CrossRef]

48. Service, USDA Foreign Agricultural. China's Maximum Levels for Contaminants in Foods. Available online: http:/ / www.webcitation.org/71ChiZoCH (accessed on 22 August 2018).

49. Awata, H.; Linder, S.; Mitchell, L.E.; Delclos, G.L. Association of Dietary Intake and Biomarker Levels of Arsenic, Cadmium, Lead, and Mercury among Asian Populations in the United States: NHANES 2011-2012. Environ. Health Perspect. 2017, 125, 314-323. [CrossRef] [PubMed]

50. Tseng, W.P. Effects and Dose-Response Relationships of Skin Cancer and Blackfoot Disease with Arsenic. Environ. Health Perspect. 1977, 19, 109-119. [CrossRef] [PubMed]

51. Tseng, W.P.; Chu, H.M.; How, S.W.; Fong, J.M.; Lin, C.S.; Yeh, S. Prevalence of Skin Cancer in an Endemic Area of Chronic Arsenicism in Taiwan. J. Natl. Cancer Inst. 1968, 40, 453-463. [PubMed]

52. Schmidt, C.W. Low-dose arsenic: In search of a risk threshold. Environ. Health Perspect. 2014, 122, A130-A134. [CrossRef] [PubMed]

(C) 2018 by the authors. Licensee MDPI, Basel, Switzerland. This article is an open access article distributed under the terms and conditions of the Creative Commons Attribution (CC BY) license (http://creativecommons.org/licenses/by/4.0/). 